\title{
Comparative study of two systems for detecting bacteraemia and septicaemia
}

\author{
N. G. WOOD \\ From the Department of Microbiology, Green Lane Hospital, Auckland, New Zealand
}

SYNOPSIS Two blood culturing systems were compared in parallel-a semi-automated radiometric method, BACTEC ${ }^{1}$, and the conventional method used in our laboratory. BACTEC contains radioactive ${ }^{14} \mathrm{C}$ labelled substrates and monitors the level of ${ }^{14} \mathrm{CO}_{2}$ evolved by actively metabolizing bacteria.

BACTEC was as efficient as the conventional method in detecting positive cultures and indicated that it was superior in detecting positive cultures earlier than the conventional system.

Rapid detection of bacteraemia is of utmost importance for patient care and prognosis. Commercial systems are available which attempt to decrease the time of detection and isolation; BACTEC is based on the detection of radioactive ${ }^{14} \mathrm{CO}_{2}$ produced by the decomposition of ${ }^{14} \mathrm{C}$ labelled glucose and other ${ }^{14} \mathrm{C}$ labelled substrates by microbial action. The semi-automated model BACTEC 301 was used in this comparative study. One hundred and thirtynine paired aerobic and anaerobic blood cultures were examined by both systems from patients at Green Lane Hospital, Auckland.

\section{Material and Methods}

Each system employs two culture bottles, one for cultivating aerobes and one for anaerobes. All bottles were inoculated with $3 \mathrm{ml}$ of blood drawn at the bedside by the clinical staff in the wards selected for this trial ( 2 cardiological and 2 surgical) and sent to the laboratory. Where requested, penicillinase or $\beta$ lactamase were added to the blood cultures.

\section{CONVENTIONAL SYSTEM}

The conventional system consisted of diphasic glucose broth containing $50 \mathrm{ml}$ of brain-heart infusion broth with $1 \%$ glucose and $0.05 \%$ sodium polyanethol-sulphonate for the aerobes, and $20 \mathrm{ml}$ thioglycollate broth with indicator-135 (Baltimore Biological Laboratories) and sodium polyanetholsulphonate for the anaerobes. An atmosphere con-

\footnotetext{
'Johnston Laboratories Inc, Cockeysville, Maryland, USA
}

Received for publication 25 November 1975 taining $5 \% \mathrm{CO}_{2}$ and $95 \% \mathrm{O}_{2}$ was added to the $\overrightarrow{0}$ glucose broth bottles. Cultures were incubated at $\sigma$ $36^{\circ} \mathrm{C}$ and examined visually twice daily for 10 days. Films were made from second day glucose broth cultures and stained with May-Grünwald/Giemsa for the presence of organisms not visually observable. Any visually suspicious or positive cultures $\stackrel{\circ}{\varnothing}$ were Gram stained and subcultured accordingly. At $\stackrel{\varrho}{\rightarrow}$ day 10 the remaining negative glucose broth cultures had films made and stained with May-Grünwald/ Giemsa and were subcultured onto blood and chocolate agar half plates. These were incubated in a candle jar at $36^{\circ} \mathrm{C}$ for 48 hours before being dis-? carded.

Anaerobic cultures were examined similarly, May-Grüwald/Giemsa stains being made and ex- $\delta$ amined on second and fifth day cultures. All day $10 ₹$ cultures and any suspicious or positive cultures were $ᄋ$ subcultured onto prereduced brain-heart infusion $\vec{D}$ agar (BBL) and incubated anaerobically, and also onto blood agar for aerobic culture. All subcultures were kept for 48 hours before being discarded.

BACTEC SYSTEM (semi-automated model 301) The BACTEC system consisted of two vials-the o aerobic vial (6A vial) containing tryptic soy broth under a $\mathrm{CO}_{2}$ atmosphere and the anaerobic vial (7B vial) containing reduced tryptic soy broth under $\stackrel{\mathbb{Q}}{+}$ a $\mathrm{N}_{2} / \mathrm{CO}_{2}$ atmosphere. Each vial contained sodium 0 polyanethol-sulphonate and ${ }^{14} \mathrm{C}$ labelled substrates $(1.5 \mu \mathrm{Ci})$.

All day 1 and day 2 aerobic cultures were placed on a reciprocating shaker in a $36^{\circ} \mathrm{C}$ waterbath and tested three times on day 1 and twice on day 2 . The remaining negative cultures were placed in an 
incubator $\left(36^{\circ} \mathrm{C}\right)$ and tested on each of the remaining five days.

The anaerobic vials were not agitated and were tested first on day 2 ; the negatives were reincubated and tested once on the following five days. All cultures were examined visually before being read on the instrument.

The atmosphere contained in the culture vials was drawn into an ionization chamber within the BACTEC 301 instrument and the amount of radioactivity was measured and displayed on an arbitrary scale, the growth index (GI) ranging from 0 to 100 . GI readings of 25 or more were considered to be positive and were immediately Gram stained and subcultured as for the conventional method.

The flushing gases used were: for the aerobic vial $5 \% \mathrm{CO}_{2} /$ air, and for the anaerobic vial $10 \% \mathrm{CO}_{2} /$ $10 \% \mathrm{H}_{2} / \mathrm{N}_{2}$ which had been passed through a DEOXO catalyst (Englehard Industries, E. Newark, $\mathrm{NJ})$ to remove any traces of oxygen.

All BACTEC vials were incubated for seven days, negative cultures being stained and subcultured at day 7 as for the conventional method.

Staphylococcus epidermidis, Bacillus species, and aerobic and anaerobic diphtheroids were considered to be contaminants unless they were growing in a number of cultures from the same patient. In these cases the significance of the isolates was determined by the patient's clinical condition.
Results

Of 139 blood cultures from 58 patients, $28(20 \cdot 1 \%)$ cultures were positive by one or both methods. Contaminants were isolated from $12(8.6 \%)$ of these, leaving $16(11.5 \%)$ positive cultures from six patients with true bacteraemia/septicaemia. Neither system detected all positive patients, each detected 14 $(88 \%$ ) positive cultures (table I). The organisms isolated by each system are given in table II. Others (Brooks and Sodeman, 1974; Smith and Little, 1974; Thiemke and Wicher, 1975) have found a lower frequency in isolating coliform organisms by BACTEC. The results tabulated in table II indicate that this could be so although the number of enteric isolates is lower.

All the Proprionebacterium acnes were isolated from one patient undergoing dental surgery and were considered significant.

An interesting isolate of a yeast, Hansenula anomala, grew in two sets of cultures from one patient with endocarditis. In the first set the conventional method was positive on day 3 compared with the BACTEC positive on day 7. The second set was positive in both systems by day 3 .

Of the 12 paired positive cultures, four $(33.3 \%)$ were detected first by BACTEC, one $(8.3 \%)$ by the conventional method, and seven $(58.3 \%)$ were detected simultaneously by each system.

\begin{tabular}{|c|c|c|c|c|c|c|c|c|}
\hline \multirow[t]{2}{*}{ Method } & \multicolumn{2}{|c|}{ Positive Cultures } & \multicolumn{2}{|c|}{ Positive Patients 1} & \multicolumn{2}{|c|}{ Organisms } & \multicolumn{2}{|c|}{ Contaminants $^{2}$} \\
\hline & No. & $\%$ & No. & $\%$ & No. & $\%$ & No. & $\%$ \\
\hline $\begin{array}{l}\text { BACTEC } \\
\text { Conventional } \\
\text { One or both methods }\end{array}$ & $\begin{array}{l}21 \\
19 \\
28\end{array}$ & $\begin{array}{r}75 \\
68 \\
100\end{array}$ & $\begin{array}{l}14 \\
14 \\
16\end{array}$ & $\begin{array}{r}88 \\
88 \\
100\end{array}$ & $\begin{array}{l}23 \\
20 \\
31\end{array}$ & $\begin{array}{r}74 \\
65 \\
100\end{array}$ & $\begin{array}{l}9 \\
5 \\
4\end{array}$ & $\begin{array}{r}64 \\
36 \\
100\end{array}$ \\
\hline
\end{tabular}

Table I Summary of BACTEC results: 139 blood cultures from 58 patients examined in parallel

${ }^{1}$ Numbers of growing cultures from positive patients.

'Some vials grew more than one contaminant.

\begin{tabular}{|c|c|c|c|}
\hline \multirow[t]{2}{*}{ Organism } & \multicolumn{3}{|l|}{ Method } \\
\hline & $B A C T E C$ & Conventional & One or both methods \\
\hline $\begin{array}{l}\text { Staph aureus } \\
\text { E. coli } \\
P . \text { acnes }^{1} \\
\text { Thiol dependent streptococcus } \\
\text { Klebsiella } \\
\text { Hansenula anomala (yeast) } \\
\text { Total }\end{array}$ & $\begin{array}{r}1 \\
1 \\
4 \\
6 \\
0 \\
2 \\
14\end{array}$ & $\begin{array}{c}1 \\
2 \\
3 \\
6 \\
12 \\
2 \\
15\end{array}$ & $\begin{array}{r}1 \\
2 \\
5 \\
6 \\
1 \\
2 \\
17\end{array}$ \\
\hline
\end{tabular}

Table II Organisms isolated from positive patients

${ }^{1}$ All were isolated from the same patient; 5 out of 9 cultures grew $P$. acnes and this was considered significant.

${ }^{2}$ One culture isolated two organisms, $E$. coli and Klebsiella.

Contaminants included $S$. epidermidis, Staph. aureus (grown in 1 out of 18 culture bottles), aerobic sporing bacillus, and aerobic and anaerobic diphtheroids. 
All organisms isolated from positive patients were detected by day 7 by each system.

Two BACTEC vials grew Proprionebacterium acnes and Staphylococcus aureus on subculture at day 7. These were considered to be contaminants: the Staph. aureus grew in one out of 18 culture bottles taken from one patient.

A number of cultures produced GI readings $>25$ with no organisms demonstrable in a May Grünwald/Giemsa film or on subculture. These were referred to as 'false positive' cultures and included $17(12.2 \%)$ of the total 139 cultures. Most of these gave a GI reading of less than 30 , seven $(5 \%)$ giving GI readings of more than 30 . An explanation of this phenomenon was that actively metabolizing leucocytes may have contributed to the false positive readings (Thiemke and Wicher, 1975). Indeed, leucocyte counts determined at the time of culturing showed that each of these patients had increased leucocyte counts ranging from 10.8 to $29.3 \times 10^{3}$ cells $/ \mathrm{mm}^{3}$ with an average count of $24.0 \times 10^{3}$ cells $/ \mathrm{mm}^{3}$. Cultures taken from two patients produced high GI readings (in excess of 50 at day 7), no organisms being demonstrated on repeated subculturing and staining. These may constitute a small proportion of cultures where organisms were present but were not demonstrable. One patient was suffering from a meningococcal meningitis with a haematological blood film picture consistent with severe infection. The other patient was admitted to hospital with abdominal pain and lobar pneumonia.

An alarming $12(75 \%)$ positive cultures were visibly growing in the anaerobic $7 \mathrm{~B}$ vial with GI readings less than 25 , only four $(25 \%)$ positive cultures producing a GI of more than 25 .

There were no 'false negative' cultures among the aerobic $6 \mathrm{~A}$ vials.

\section{Discussion}

The data presented indicated that the BACTEC system was as efficient as our routine method in isolating organisms from bacteraemic/septicaemic patients. BACTEC also appeared to be superior in detecting positive cultures earlier than our conventional method. There is general agreement with the results of this study and results obtained by others (Brooks and Sodeman, 1974; Thiemke and Wicher, 1975). However, it must be stressed that the total number of cultures examined and the number of positive cultures detected was small. We were supplied with a limited amount of media for this initial comparative study and were unable to obtain further supplies in order to assess a larger range of patients.

From previous experience in this laboratory, Giemsa staining was found to be superior in de- tecting small numbers of Gram-negative organisms in blood cultures which otherwise may have been easily missed in a Gram stain. Any positive Giemsa films were subsequently Gram stained and subcultured onto appropriate media.

A major concern was the occurrence of the false positive cultures. The number of false positive and false negative cultures varied, depending upon what on GI would be taken as showing the presence of $\vec{\circ}$ viable organisms. In this study a GI of 25 or more was taken as an indication of growth, giving an incidence of $12.2 \%$ false positive cultures, all occurring in the aerobic $6 \mathrm{~A}$ vial. There were no false negatives among the aerobic $6 \mathrm{~A}$ vials but $75 \%$ of the positive anaerobic culture vials (7B vials) failed to register a positive GI reading. This emphasizes the need for visually checking cultures for signs of growth before reading on the instrument. Several factors may have contributed to the high incidence of false negative anaerobic cultures:

(1) Inability of the organism to produce ${ }^{14} \mathrm{CO}_{2}$ from the substrates supplied. This would indicate an expansion of the ${ }^{14} \mathrm{C}$ substrates provided in the media by the manufacturers, to accommodate these organisms.

(2) Differing modes of metabolism under anaerobic conditions. The thiol dependent streptococcus failed to register a positive GI reading in the anaerobic vial although they were visually growing, yet in the aerobic vial they were positive on the BACTEC before there was visible sign of growth. Most of the $P$. acnes isolated and two contaminating aerobic sporing bacilli also failed to register positive GI readings.

(3) The anaerobic vials had expired their suggested period of use just before the beginning of this study and a further supply could not be obtained.

Brooks and Sodeman (1974) used a GI reading of $\frac{\circ}{3}$ 20 or greater with an incidence of $43 \%$ false positive cultures. They suggested that if a GI reading of $30 ?$ had been used, the number of negative vials sub- $\frac{D}{2}$ cultured would have been $1.5 \%$ at the expense of larger detection times for $95 \%$ of the positive $\sigma$ Candida cultures. These generally gave a GI reading N of less than 30 at the time of subculture.

Others (Smith and Little, 1974; Thiemke and ${ }_{\sigma}^{\omega}$ Wicher, 1975) have used a GI reading of 30 as indicative of growth. Thiemke and Wicher (1975) found $6.8 \%$ false positive cultures overall; however, $\stackrel{\$}{\rightarrow}$ $6.8 \%$ of the positive cultures failed to register a positive GI. Of 14 isolates in this category, nine were fungi. Smith and Little (1974) found $8.9 \% \stackrel{\mathbb{D}}{\mathbb{D}}$ false positive cultures in their study, $6.3 \%$ positive $\frac{\rho}{\mathbb{Q}}$ cultures giving a GI of less than 30 .

After examining these studies we decided to compromise and take 25 as a positive GI reading. 
It became apparent that several organisms, although present in the BACTEC or conventional vials, failed to grow on subculture. This emphasized the need to be aware of their inability to grow on ordinary subculturing media and for designing a suitable medium for their cultivation. These organisms included the thiol dependent streptococcus and many of the anaerobic diphtheroids. Solid media for subculturing were improved by incorporating Lcysteine, menandione, haemin, and yeast extract in brain-heart infusion agar. Dithiothereatol and palladium chloride were added to the supplemented BHI agar and pre-reduced for the isolation of fastidious anaerobes.

Subculturing techniques must be of a high quality so that organisms found on primary blood culture can be subcultured for further studies (ie, identification and susceptibility testing). A good knowledge and high degree of expertise is essential for blood culture work. The BACTEC 301 semi-automated instrument, although removing the need for multiple staining procedures, does not alleviate the tedium of testing large numbers of cultures. In our small trial one person was committed to sampling for most of the day, and if all blood cultures were tested the BACTEC 301 would be unsuitable. In considering an automated method for blood cultures it would be necessary to use the fully automated BACTEC 225, which would allow the technologist freedom to give full attention to subculturing and testing positive cultures.

A further trial of at least 500 patients throughout the whole hospital is considered necessary to do justice to this type of equipment (preferably the 225).

No system has yet been devised that will detect all bacteraemic/septicaemic patients. Although this study involved only a small number of cultures the results indicated that BACTEC is equal to our conventional method in detecting bacteraemia/septicaemia.

I should like to thank Mr B. Cornere for his advice and assistance in the presentation of this paper.

\section{References}

Brooks, K. and Sodeman, T. (1974). Rapid detection of bacteremia by a radiometric system: a clinical evaluation. Amer. J. clin. Path., 61, 859-866.

Smith, A. G. and Little, R. R. (1974). Detection of bacteremia by an automated radiometric method and a tubed broth method. Ann. clin. Lab. Sci., 4, 448-455.

Thiemke, W. A. and Wicher, K. (1975). Laboratory experience with a radiometric method for detecting bacteremia. J. clin. Microbiol., 1, 302-308. 